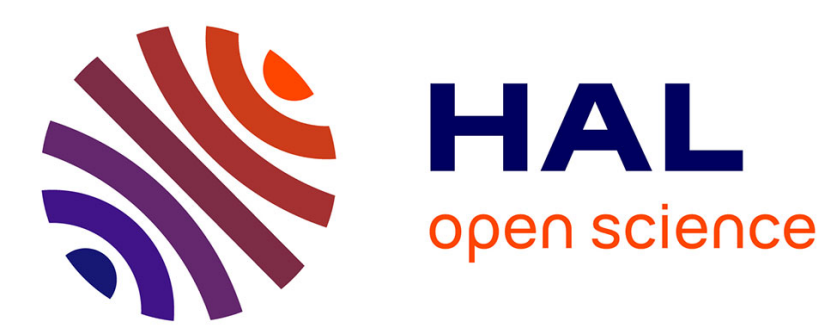

\title{
L'eau comme enjeu de pouvoir : l'exemple de Tianjin dans la première moitié du XX e siècle
}

Delphine Spicq

\section{To cite this version:}

Delphine Spicq. L'eau comme enjeu de pouvoir : l'exemple de Tianjin dans la première moitié du XX e siècle. Études Chinoises, 2005, 24, pp. 137-158. 10.3406/etchi.2005.1355 . hal-02394482

\section{HAL Id: hal-02394482 \\ https://hal.science/hal-02394482}

Submitted on 4 Dec 2019

HAL is a multi-disciplinary open access archive for the deposit and dissemination of scientific research documents, whether they are published or not. The documents may come from teaching and research institutions in France or abroad, or from public or private research centers.
L'archive ouverte pluridisciplinaire HAL, est destinée au dépôt et à la diffusion de documents scientifiques de niveau recherche, publiés ou non, émanant des établissements d'enseignement et de recherche français ou étrangers, des laboratoires publics ou privés. 


\section{L'eau comme enjeu de pouvoir : l'exemple de Tianjin dans la première moitié du $\mathrm{XX}^{\mathrm{e}}$ siècle}

\section{Delphine Spicq *}

L'eau est un élément vital pour les êtres humains. Dans un monde où cette ressource est, pour des raisons variées (augmentation de la population, changement de modes de vie et de production, pollution, désertification, etc.), accessible en quantités de plus en plus faibles, son enjeu va sans aucun doute constituer l'une des principales sources de conflits entre États. Cette situation n'est certes pas nouvelle : contrôler l'eau est acquérir du pouvoir car il s'agit d'une ressource économique primordiale. Inversement, le pouvoir politique et militaire local d'un groupe particulier détermine son accès à l'eau.

Ce petit article propose d'illustrer ce thème par l'exemple de la ville de Tianjin entre la fin du $\mathrm{XIX}^{\mathrm{e}}$ et le milieu du $\mathrm{XX}^{\mathrm{e}}$ siècle. En quoi et pourquoi l'approvisionnement en eau de cette ville a-t-il été un enjeu de pouvoir ? Plus précisément, en quoi la maîtrise de la ressource et le contrôle des sociétés d'adduction d'eau ont-ils contribué à accroître le pouvoir politique et économique de certains groupes sociaux ?

D’une façon générale, on assiste sur toute la période concernée à une lutte pour le contrôle de l'eau entre Chinois mais aussi entre Chinois et étrangers à partir de l'installation de ces derniers dans des concessions à

\footnotetext{
* Institut des Hautes Études Chinoises, Collège de France.
} 


\section{Delphine Spicq}

proximité de la ville chinoise. Les oppositions sont multiples : municipalité chinoise contre conseils municipaux des concessions, citadins contre ruraux, marchands contre mandarins et compagnies des eaux contre administrations.

Tianjin en 1900

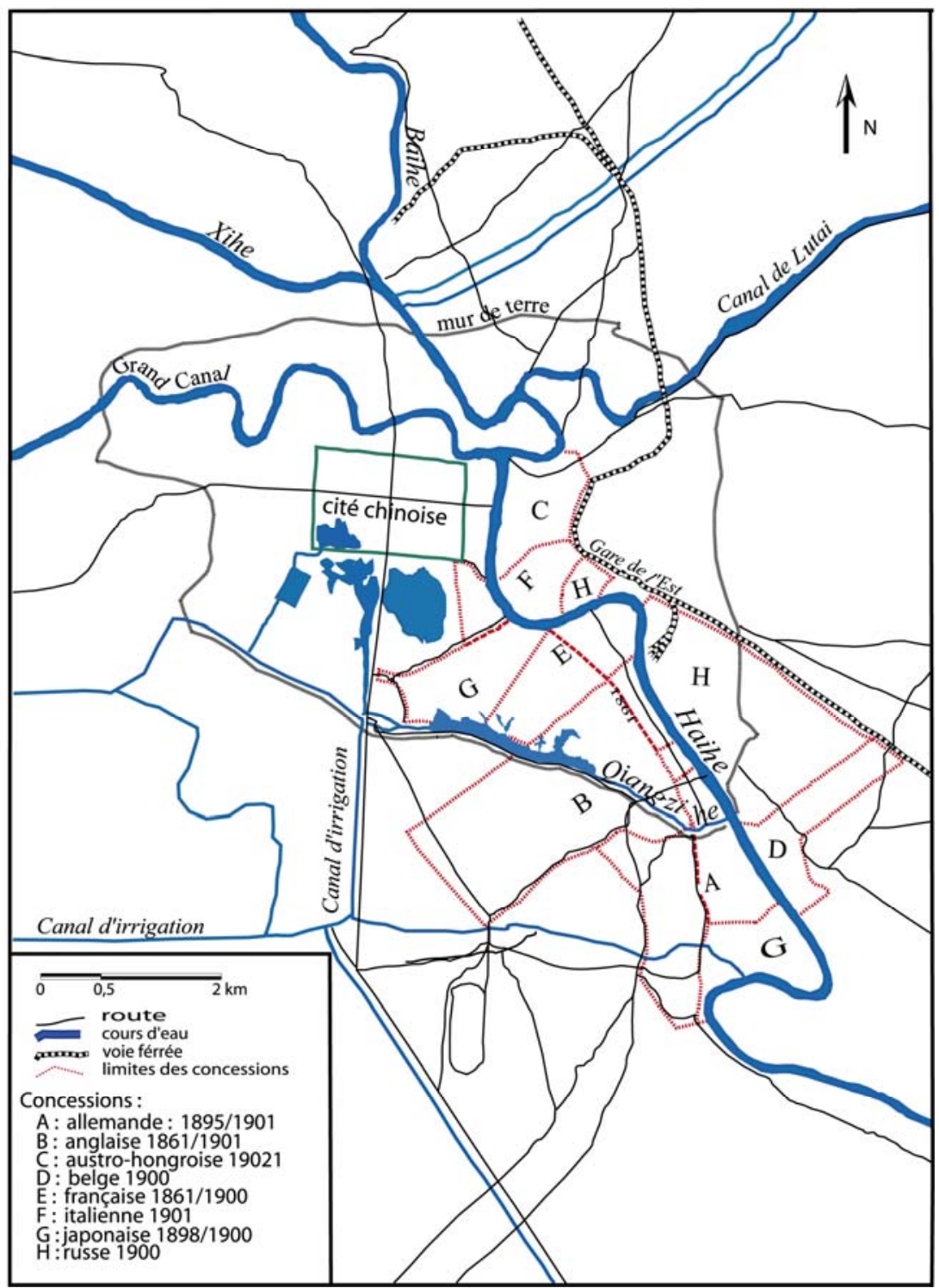

Carte du réseau hydrographique de la région de Tianjin en 1900

Source : Archives du ministère des Affaires étrangères, Paris, Correspondance politique et commerciale, Nouvelle série, Chine, 1897-1917, vol. 287. 
Cette lutte pour la maîtrise de l'approvisionnement de l'eau a pris une importance particulièrement aiguë à la suite d'évènements politiques graves tels que l'insurrection des Boxeurs et la fin de la seconde guerre mondiale. Il faut ajouter à ces évènements des préoccupations écologiques : diminution continue des quantités disponibles du fait d'une pollution croissante et d'une hausse soutenue de la consommation et processus écologiques de long terme - alluvionnement des cours d'eau, assèchement des sols, crues récurrentes.

Tianjin, ville située dans la Plaine du Nord, à une centaine de kilomètres au sud-est de Pékin, a toujours connu des difficultés à accéder à l'eau et à la maîtriser du fait de sa situation géographique. Bien que traversée par plusieurs cours d'eau, au contraire de sa voisine la capitale, la ville a alternativement connu inondations ou sécheresses selon les fantaisies du climat ${ }^{1}$. En outre, les grandes rivières qui irriguent la plaine autour de Pékin et Tianjin et qui confluent dans le Haihe 海河 (voir carte) se caractérisent toutes par des débits très fluctuants et des crues rapides et violentes que des siècles de maîtrise hydraulique n’ont pas réussi à dompter.

\section{La ville}

$\mathrm{Au} \mathrm{XIX}$ siècle, et probablement avant, le niveau d'étiage très bas du Grand Canal à certaines époques de l'année pose un problème crucial : un niveau minimum doit être maintenu afin d'assurer le transport du Tribut en grain vers Pékin. Or, le développement d’une agriculture locale fondée sur la riziculture exige une irrigation importante et donc des prélèvements considérables dans le Canal. Entre ces deux usages concurrents, c'est

\footnotetext{
${ }^{1}$ L'absence d'un cours d'eau à l'intérieur de la ville a toujours handicapé Pékin et a très tôt imposé la construction de canaux. Cette configuration particulière a préservé la ville des inondations, en partie seulement car les crues indomptables du Yongdinghe 永定河 ont nécessité la construction de digues à l’ouest pour protéger la ville.
} 


\section{Delphine Spicq}

l'alimentation de la capitale en grain qui a toujours été privilégiée. La croissance de la ville et les besoins en eau qui en résultent aggravent encore la situation.

Vers 1850, Tianjin est en effet une cité de premier ordre : avec plus de 100000 habitants elle compte parmi les villes les plus peuplées de l'Empire (avec Pékin, Canton et Shanghai). Petite garnison au début des Ming (1368-1644), elle a vu son commerce croître rapidement du fait de sa position géographique favorable. Située près de la mer, elle joue un rôle de lien naturel entre les côtes et l'intérieur du pays. Mais elle se trouve aussi à la confluence de nombreux cours d'eau, dont le plus important au niveau économique et politique est le Grand Canal ; elle est enfin, de par sa position à la croisée des principales routes commerciales interrégionales entre le Nord et le Sud, devenue le principal entrepôt de la Chine du Nord. Les marchandises qui transitent par ses quais et ses entrepôts ou qui y sont vendus proviennent de Mongolie, de Mandchourie, de l'intérieur du Hebei ou de provinces du Sud comme le Zhejiang ou le Jiangsu.

La production et la commercialisation du sel ont aussi contribué à l'essor d'un commerce florissant et très ancien. L'État en tire profit dès les Ming, lorsque l'empereur Wanli 萬歷 (r. 1573-1620) impose une taxe commerciale pour financer la reconstruction du Palais impérial ${ }^{2}$ et y installe une partie de l'administration de la gabelle (changlu 長蘆) qui a la charge de la commercialisation du sel dans la région. Au début des Qing (1644-1911), un poste des douanes maritimes puis le Bureau de supervision de la gabelle sont installés dans la ville. Cette importance commerciale et financière grandissante se traduit par une promotion politique lorsque l'empereur Yongzheng 雍正 (r. 1723-1735) fait de cette petite sous-préfecture une préfecture de premier rang (fu 府) en 1731.

\footnotetext{
${ }^{2}$ Kwan Manbun, The salt merchants of Tianjin state-making and civil society in late imperial China, Honolulu : University of Hawai’i Press, 2000, p. 17.
} 


\section{La question de l'eau}

Le système d'approvisionnement en eau qui prévaut à Tianjin avant l'arrivée des Occidentaux est celui que l'on retrouve dans la plupart des grandes villes avant l'avènement de circuits de distribution modernes. La population s'approvisionne aux sources disponibles localement : les cours d'eau, principalement le Grand Canal ou Nanyunhe 南運河 et, dans une moindre mesure, le Haihe ainsi que les puits. Elle va chercher l'eau ellemême ou bien loue le service de porteurs-vendeurs. L'approvisionnement est un souci quotidien pour les habitants pour plusieurs raisons : les cours d'eau étant éloignés de la cité murée, on se procure le précieux liquide le plus souvent auprès de porteurs, généralement liés à la pègre locale, qui sont querelleurs et peu honnêtes. On ne peut cependant pas se passer d'eux, puisqu'ils contrôlent la distribution. Seule l'eau des puits, pour la plupart privés, échappe à leur contrôle, mais elle ne représente qu'une faible part de la consommation totale ${ }^{3}$. Ils pratiquent des prix élevés qui peuvent représenter jusqu'à la moitié de la somme quotidienne nécessaire pour nourrir une personne ${ }^{4}$. De plus, les méthodes de transport rudimentaires cruches, seaux, récipients creux de toutes sortes, palanches, brouettes et charrettes - ne permettent qu'un approvisionnement en faibles quantités. Enfin, il faut encore traiter l'eau avant de l'utiliser. Le traitement le plus courant consiste à la faire décanter dans de grandes jarres avec un précipité à base d'alun (sulfate d'alumine). Les diverses particules présentes dans l'eau sont entraînées au fond du récipient puis évacuées à l'aide d'un tuyau en bambou. Il faut encore bouillir l'eau avant de la boire. Certains ajoutent même une étape et versent des haricots rouges ou des grains de sésame

${ }^{3}$ Delphine Spicq, L'hydraulique urbaine et la politique de l'eau dans la Plaine du Nord de la Chine, le cas de Tianjin, 1900-1949, Thèse de $3^{\mathrm{e}}$ cycle, Paris : Université Paris VII - Denis Diderot, UFR LCAO, 2003, p. 60.

${ }^{4}$ D. Spicq, ibid., p. 63, donnée tirée de Wu Tinghua 吳廷華, Tianjin xianzhi 天津縣志 (1739), Réimpression [1 ${ }^{\text {re }}$ impression 1739]. Keben 1870, j. 5, p. 23a. 


\section{Delphine Spicq}

qu'on laisse reposer plusieurs jours dans la jarre. Ils sont censés débarrasser l'eau des bactéries ${ }^{5}$.

L'arrivée des étrangers à Tianjin va progressivement modifier la ville dans sa structure, son organisation et son fonctionnement. Curieusement oubliée dans le Traité qui porte son nom, signé entre les Puissances et le gouvernement chinois en 1858, la ville est finalement ouverte au commerce et l'installation des étrangers y est autorisée en 1860 à la suite de la signature de la Convention de Pékin. La présence étrangère est dans un premier temps faible et circonscrite aux quelques rues qui constituent les premières concessions, bâties sur des terrains situés à bonne distance de la cité chinoise et où, peu nombreux, ils vivent entre eux. Des aménagements publics rudimentaires ainsi qu'une administration et des services municipaux (voirie, ramassage des ordures, éclairage public, services de police et de santé publique) sont mis en place, assez tardivement, vers la fin des années 1870. Les administrations ainsi créées et les différents services qui les composent jouent un rôle déterminant puisqu'ils vont servir de modèle lors de la création d'une administration moderne pour la ville chinoise, et en particulier pour le système d'alimentation en eau de la ville.

\section{Les premières modernisations}

Les étrangers s’installant peu à peu dans les concessions à partir des années 1870 ne sont pas satisfaits des différentes méthodes auxquelles ils doivent recourir pour se procurer de l'eau, ni de sa qualité. Des discussions animées, portant sur le meilleur système d'adduction à mettre en place, ont lieu dès le milieu des années 1870 au sein de la communauté étrangère. Les désaccords concernent d'abord le choix de la ressource, cours d'eau ou

\footnotetext{
${ }^{5}$ Ruth Rogaski, From protecting life to defending the Nation. The emergence of public health in Tianjin 1859-1953, PhD. dissertation, New Haven : University of Yale, 1996, p. 37-38.
} 
puits, puis le mode de gestion, public ou privé, de la future société. Dans une troisième phase, les différents conseils municipaux des concessions, poussés par la rivalité, essaient, dans la hâte, de faire aboutir leur projet en premier. Les Français foreront, sans succès, le sous-sol de leur concession.

Il faudra attendre les dernières années du $\mathrm{XIX}^{\mathrm{e}}$ siècle pour que les étrangers se décident à mettre en place un système de distribution moderne. C'est finalement les Britanniques qui créent la première compagnie des eaux de la ville en 1897, la Tientsin Waterworks Company (ci-après TWC). Les débuts sont timides : la compagnie n'alimente que la zone anglaise ${ }^{6}$. Le reste de la ville et les autres concessions continuent de dépendre du système traditionnel.

Au printemps 1900, l'insurrection des Boxeurs puis l'occupation de la ville pendant deux années par un corps expéditionnaire étranger accélèrent la transformation de la cité chinoise en une ville moderne. À Tianjin, les Boxeurs ou Yihetuan 義和团 - «Milices de justice et de concorde » sont présents dans la ville et ses environs dès le mois de mars $1900^{7}$. Ils occupent la cité chinoise à partir du début du mois de juin puis la saccagent et attaquent les concessions vers le $15^{8}$. Les étrangers sont encerclés et bombardés par les milices auxquelles s'est jointe l'armée régulière chinoise. Un corps expéditionnaire international regroupant les armées de huit pays (Allemagne, Autriche-Hongrie, France, Grande-Bretagne, États-Unis, Italie, Japon et Russie) débarque fin juin à Dagu 大沽, un des ports de Tianjin sur le Bohai. Il libère les concessions puis attaque et occupe la cité murée, avant d'aller délivrer les légations de Pékin (qui ne sont effectivement libérées que le 15 août). Une semaine plus tard, un gouvernement

${ }^{6}$ J'utilise ici le terme anglaise pour britannique. L'usage de l'époque incluait dans ce terme les différentes nations britanniques.

${ }^{7}$ Tianjinshi difangzhi bianxiu weiyuanhui 天津市地方志編修委員會, Tianjin jianzhi 天津簡志, Tianjin : Tianjin renmin chubanshe, 1991, p. 1391.

${ }^{8}$ Otto Durham Rasmussen, Tientsin, an illustrated outline history, Tientsin : Tientsin press, 1925, p. 131. 


\section{Delphine Spicq}

d’occupation est créé, le Gouvernement provisoire de Tianjin. Cette instance, établie et contrôlée par le corps expéditionnaire, administrera la cité chinoise pendant deux ans, jusqu'en août 1902. Elle s'emploie à ramener l'ordre et le calme, ce qui passe en premier lieu par une amélioration de l'hygiène. Les innombrables morts et blessés ainsi que le manque d'eau et de nourriture font craindre l'apparition d'épidémies au sein de la population qui revient peu à peu dans la ville. Dans un second temps, on met en place une administration moderne dans la cité chinoise et on adopte des réformes institutionnelles. Les changements sont importants et vont jusqu'à une transformation physique de la ville : destruction des remparts de la ville murée pour faire place à un boulevard circulaire. Enfin, on encourage la création de plusieurs sociétés privées de services publics et, dans les quatre années qui suivent, des entreprises de tramway, d’électricité, de téléphone et d'adduction d'eau voient le jour ${ }^{9}$.

Lorsque Yuan Shikai 袁世凱 (1859-1916), le nouveau gouverneur de la province du Zhili, se voit remettre l'administration de la ville à l'été 1902, celle-ci est devenue une cité moderne, dotée, entre autres, d'une police municipale et d'un service de santé publique. Dans le même temps, les concessions sont passées de cinq à huit, les pays membres du corps expéditionnaire qui en étaient dépourvus ayant profité de l'occasion pour s'en octroyer une. Tianjin détient alors le record du nombre de concessions, et devient, pour reprendre l'historienne Ruth Rogaski ${ }^{10}$, une «hypercolonie ».

La création de la seconde société d'adduction s’inscrit donc dans un contexte différent de celui de la première. Le projet fait partie intégrante du

${ }^{9}$ La Compagnie du tramway et de l'électricité, société belge qui administre les tramways de la ville, est fondée en 1904, la Compagnie du téléphone de Tianjin est créée en 1906 ; cf. Tianjin jianzhi, p. 320-329.

10 Ruth Rogaski, “Hygienic modernity in Tianjin”, in Remaking the Chinese city, modernity and national identity 1900-1950, Honolulu : University of Hawai'i Press, 2000, p. 34. 
plan de modernisation de la ville chinoise. Il est au départ porté par des officiels et des marchands chinois proches des milieux étrangers et du Gouvernement provisoire. Les trois principaux initiateurs sont Chen Jiyi 陳 濟易 (s. d.), Ma Yuqing 馬玉清 (s. d.) et Rui Yukun 苁玉坤 (s. d.). Le premier est le fondateur de la Shanghai Waterworks (compagnie des eaux de la cité chinoise de Shanghai qui démarre son activité peu de temps avant, en 1900) ; le second est un compradore au service d'une entreprise étrangère de fabrication de kérosène et d'allumettes de Tianjin et le troisième est un marchand travaillant pour le compte d'une société mixte d'importexport de Tianjin également ${ }^{11}$.

Ces trois hommes sont bien introduits dans les milieux étrangers et connaissent personnellement le secrétaire du Gouvernement provisoire de Tianjin, un diplomate américain du nom de Charles Denby. C'est sur son conseil qu'ils entament des démarches auprès du Gouvernement provisoire pour fonder la Tientsin Native City Waterworks Company, Ji'an zilaishui gongsi 濟安自來水公司 (ci-dessous TNCWC), financée en majeure partie par des capitaux non chinois car, à Tianjin comme dans d'autres villes chinoises à l'époque, l'épargne privée indigène est insuffisante ${ }^{12}$. La technologie, le matériel et le savoir-faire sont également d'origine étrangère. C'est en fait dans ce domaine particulier que la dépendance de la Chine à l'égard de l'étranger est la plus marquée, car il n'existe pas à l'époque de personnel technique chinois compétent pour faire fonctionner un matériel conçu et fabriqué à l'étranger.

Nous avons donc la création d'une seconde société d'adduction d'eau qui, comme la première, est en réalité aux mains des étrangers, avec la bénédiction du Gouvernement provisoire qui accepte la «direction » d'amis et de relations chinois. Le corps expéditionnaire, qui fait de la lutte

${ }^{11}$ Wang Huatang 王华棠, Tianjin, yi ge chengshi de jueqi 天津, 一个城市的崛起, Tianjin : Tianjin renmin chubanshe, 1990, p. 170-171.

${ }^{12}$ D. Spicq, op. cit., p. 107-108. 
contre les épidémies et l'instauration d'un ordre sanitaire sa priorité, place la ressource en eau et la distribution au centre de son action. Son contrôle a un but politique mais aussi, bien évidemment, économique : l'adduction d'eau est déjà à l'époque un secteur d'activité très rentable, ce que les commerçants et financiers qui investissent dans la société d'adduction n’ignorent pas.

La TNCWC alimente l'ancienne cité murée, les quartiers chinois et quelques concessions (trois sur huit en 1906, davantage par la suite). À cette date, elle a une clientèle d'environ 150000 personnes soit dix fois plus que la clientèle de la société de la concession anglaise qui n’approvisionne alors que trois concessions. La différence de taille entre les deux populations desservies illustre le rôle distinct dévolu à chacune de ces deux sociétés : la TWC ne s'adresse qu'aux étrangers des concessions alors que la TNCWC approvisionne la majeure partie des habitants (tous les quartiers chinois et plusieurs concessions).

Aux enjeux politiques et économiques s'ajoute rapidement une dimension symbolique. La concession des différents services publics de la cité chinoise à des sociétés non chinoises par le Gouvernement provisoire est fortement critiquée par les notables de Tianjin lors de la rétrocession de l'administration de la cité en août $1902{ }^{13}$.

Pourtant, les habitants de l'ancienne cité chinoise se mettent à utiliser l'eau de la TNCWC et prennent peu à peu conscience de l'utilité, voire de la nécessité de ce service, puis dans un second temps du pouvoir que cette activité confère à la société sur la population et sur l'activité économique. C’est une fois évaluée l'étendue du pouvoir généré par le monopole de la production et de la distribution d'eau que le système d'adduction devient un enjeu entre les différentes composantes sociales, économiques et politiques de la ville.

${ }^{13}$ Archives du ministère des Affaires étrangères, Paris, Correspondance politique et commerciale, $\mathrm{N}^{\text {lle }}$ série, Chine, 1897-1917, vol. 287, lettre du ministre de France à Pékin au ministre des Affaires étrangères à Paris, en date du 30 octobre 1902. 
La lutte pour le contrôle de l'eau revêt alors différentes formes : remise en question du contrôle des sociétés par les étrangers, tentative de reprise en main de la politique de l'eau par l'administration chinoise et, surtout, lutte pour la maîtrise de deux éléments essentiels de la chaîne de distribution de l'eau, l'accès à la ressource et le contrôle de sa distribution.

\section{Eau, politique et économie}

L'évolution de la structure du capital de la TNCWC illustre la lutte que se livrent les différentes entités politiques et économiques ainsi que divers groupes sociaux pour le contrôle du système d'adduction. Au moment de sa création, cette société a un capital certes plus diversifié que la compagnie de la concession anglaise, la TWC, mais surtout beaucoup plus important en taille et en capacité de production ${ }^{14}$. L'actionnariat étranger est majoritaire avec environ $60 \%$ du total, ce qui explique la suprématie des étrangers sur le conseil d'administration et la direction de la société. Par ailleurs, il semble que cet actionnariat soit essentiellement composé de marchands, industriels et hommes d'affaires, mais également de quelques sociétés privées. Malgré une part de l'actionnariat chinois en augmentation constante pendant les décennies 1910-1920, on n'observe pas de remise en cause de la mainmise étrangère, occidentale principalement, jusqu'en 1936. À cette date, les Chinois détiennent plus de $70 \%$ du capital ${ }^{15}$ et réclament une représentation plus importante au sein du conseil d'administration, encore majoritairement composé d'étrangers, ainsi que la désignation d'un directeur de nationalité chinoise.

\footnotetext{
${ }^{14}$ Le capital de départ de la TNCWC est de 800000 taëls réparti en 8000 actions, l'autre société a un capital initial de 65000 taëls en 650 actions. Voir D. Spicq, op. cit., p. 147-148.

${ }^{15}$ Tianjin tebie shi gongshu gongyong chu 天津特別市公署公用處, Xingzheng gongzuo ji shiye jihua 行政工作及事業計劃, Tianjin : 1941, p. 56.
} 
Tous les secteurs d'activité, de services ou de production ont besoin d'eau, de l'entreprise individuelle de coiffure au forgeron, a fortiori les industries nouvelles de la ville telles que la fabrication et la réparation de machines-outils, l'imprimerie, les filatures, la chimie ${ }^{16}$, etc. La politique de la société d'adduction en termes de prix, de méthode de traitement de l'eau, d'approvisionnement et de distribution, a donc des répercussions aussi bien au niveau macroéconomique que microéconomique. Une représentation majoritaire des marchands et industriels chinois au conseil d'administration leur permettrait d'influer directement sur les choix futurs de la société en matière de développement de l'activité, de prix, d'extension du réseau, d'orienter cette politique dans un sens favorable et de renforcer ainsi leur pouvoir politique et économique sur la ville, au détriment d'autres catégories sociales ou économiques. Cette politique d'acquisition n'est certes pas limitée à l'adduction d'eau, et concerne aussi le capital des sociétés jouant un rôle de premier plan dans la vie économique de la cité.

Tandis que les hommes d'affaires chinois s'efforcent d'accroître leur actionnariat dans les entreprises, les compagnies des eaux sont également l'enjeu de luttes entre les deux entités politiques principales de la ville : le gouvernement municipal chinois et les différentes municipalités des concessions. Mais leurs raisons sont cependant sensiblement différentes : l'adduction d'eau constitue pour chaque municipalité un des élément majeurs de sa politique de santé publique et de bien-être de sa population. Mais c'est aussi, quoique dans une moindre mesure, un élément de sa politique économique. Les différentes municipalités tentent d'infléchir les décisions de la société d'adduction et interviennent même de façon directe, en lui imposant leurs desiderata. C'est le cas de la municipalité de la concession japonaise qui, en 1916, exige qu'un de ses représentants siège

\footnotetext{
${ }^{16}$ Tianjinshi difangzhi bianxiu weiyuanhui 天津市地方志編修委員會, Tianjin tongzhi -, fuzhi - zujie 天津通志 Tianjin : Tianjin shehui kexueyuan chubanshe, 1996, p. 195-205.
} 
au conseil d'administration de la TNCWC qui approvisionne sa concession. Sa dépendance en matière d'adduction d'eau par rapport à la compagnie lui semble un motif suffisant pour justifier cette revendication ${ }^{17}$. De son côté, le conseil municipal de la concession anglaise diminue sa dépendance par rapport à la TWC en lui imposant sa supervision directe. La société est rachetée par les autorités de la concession et devient une régie municipale au début des années $1920{ }^{18}$. Ce passage d'un statut privé à un statut public n’a pourtant pas les effets escomptés : les quantités augmentent mais de façon insuffisante, la pression dans les canalisations est trop faible et la qualité de l'eau reste incertaine. La régie remédie en partie au problème en optant pour une alimentation par des puits à partir de $1926^{19}$.

La municipalité de la ville chinoise décide quant à elle de s’affranchir de sa dépendance par rapport à la TNCWC en créant une régie des eaux dans l'ex-concession allemande - rebaptisée Premier district spécial après sa rétrocession à la Chine en 1917 - au début des années 1930. Ce quartier était jusque là alimenté par la régie anglaise. À la fin des années 1920, cette dernière ne souhaitant pas poursuivre l'approvisionnement de ce quartier, la municipalité chinoise, qui ne semble pas vouloir dépendre non plus de la TNCWC, opte pour un système d'adduction autonome pour ce quartier. Ce premier essai de société des eaux, alimentée par des puits, entièrement conçue par des Chinois ${ }^{20}$ est un échec : mal gérée,

${ }^{17}$ D. Spicq, op. cit., p. 140.

${ }^{18}$ La municipalité avait déjà tenté par le passé de racheter la société, l’ensemble des contribuables de la concession n'étant pas satisfait du service rendu. En effet, les quantités distribuées stagnaient, la qualité de l'eau n’était pas toujours irréprochable bien que son prix fût supérieur à celui de l'autre société d'adduction.

${ }^{19}$ D. Spicq, op. cit., p. 222-224. Peu après sa mise en régie, la TWC perd deux de ses clients : la concession française puis l'ex-concession allemande. De fait, à partir de la fin des années 1920, elle ne distribue d'eau qu'à la concession anglaise, et cela jusqu’à la rétrocession de celle-ci aux autorités chinoises en 1947.

${ }^{20}$ J'insiste sur le fait que la conception et la gestion sont chinoises, car la réalisation proprement dite, dont le forage des puits, est confiée à une société anglaise, aucune 


\section{Delphine Spicq}

elle est rachetée au bout de deux ans par la TNCWC et intégrée à son système de distribution.

La TNCWC prenant de plus en plus d'importance, les autorités des différentes concessions, à l'exception de la zone anglaise, vont tenter, à partir du milieu des années 1920, de remettre en cause son autonomie. Elles vont exiger un droit de regard de plus en plus important sur ses activités et sur son organisation en la menaçant régulièrement de la soumettre à de nouvelles taxes ou en édictant de nouveaux règlements, lois ou normes contraignantes.

À la part croissante de l'actionnariat chinois dans les sociétés et au contrôle grandissant de l'administration vient se greffer le problème de la sécurisation de la ressource. On a vu qu'il existait un conflit entre les exigences de la navigation sur le Canal et celles de l'irrigation. Il vient s'y ajouter, à partir du début du $\mathrm{XX}^{\mathrm{e}}$ siècle, un troisième type de besoin : l'approvisionnement en quantités importantes d'eau par le système modernisé de l'adduction. L'accès et le contrôle de la ressource deviennent alors des enjeux de pouvoir encore plus considérables et qui dépassent souvent le simple cadre local.

Le territoire placé sous l'administration directe du Gouvernement provisoire de Tianjin débordant largement les simples limites de la ville et incluant de ce fait la source à laquelle la TNCWC s'approvisionne ${ }^{21}$, le contrôle de la ressource par les Occidentaux est complète. Mais ce contrôle s'érode lentement après le départ du corps d'occupation étranger en septembre 1901, à la suite de la signature à Pékin du Protocole Boxeur. À partir du milieu des années 1910, la société se plaint régulièrement de prélèvements effectués dans le Canal, en amont de la ville, à des fins d'irrigation. Elle tente à plusieurs reprises de faire modifier la règle de répartition à son profit, sans obtenir gain de cause et se résout finalement à

compagnie chinoise n'étant à l'époque en mesure de réaliser les forages ; cf. D. Spicq, op. cit., p. 285-287.

${ }^{21}$ Il s’agit du Grand Canal. 
installer en 1921 une autre prise d'eau sur le Xihe, cours d'eau situé au nord du Grand Canal (cf. carte).

À ce conflit d'usage vient s'ajouter le problème du transport des troupes des seigneurs de la guerre. Les généraux Zhu Yupu 褚玉璞 (18871931) et Zhang Zongchang 張宗昌 (1881-1932), chefs de guerre de la clique du Zhili, préparent la retraite de leurs troupes vers le Nord-Est face à l'avancée de l'armée de Yan Xishan 閻錫山 (1883-1960), seigneur du Shanxi rallié au Guomindang dans la reconquête du territoire national (Beifa 北伐). C'est dans ces circonstances, en mai-juin 1928, qu'une vanne est ouverte à plusieurs reprises sur le Grand Canal, toujours en amont de la ville, à des fins militaires. Des quantités d'eau très importantes (environ 9/10 de l'eau disponible dans le Canal) sont soustraites à l'exploitation de la TNCWC, provoquant des ruptures d'approvisionnement.

La société a donc perdu en grande partie, vers la fin des années 1920, sa capacité de sécuriser son approvisionnement, et sa nationalité étrangère $^{22}$ et les appuis qu'elle peut obtenir auprès du corps diplomatique occidental à Tianjin ou à Pékin ne lui sont pas d'un grand secours. Cet exemple illustre l'importance du contrôle territorial, et on va en avoir plus loin une confirmation avec le cas des porteurs d'eau.

\section{La distribution}

La distribution de l'eau à l'intérieur de la ville est en effet un autre lieu de conflit de pouvoir. Le phénomène n'est pas nouveau : avant l'arrivée des sociétés d'adduction, les porteurs d'eau se livraient déjà une lutte sans merci. À partir du début du $\mathrm{XX}^{\mathrm{e}}$ siècle, reconvertis en tenanciers de bornes-fontaines au service des deux sociétés des eaux, ils s’emploient à rega-

\footnotetext{
${ }^{22}$ Enregistrée à Hong Kong, la société est de nationalité anglaise jusqu’en 1936.
} 


\section{Delphine Spicq}

gner l'ascendant qu'ils avaient autrefois sur la population des quartiers qu'ils desservaient.

Avant la modernisation, comme on l'a dit plus haut, les porteurs allaient chercher l'eau aux rivières et aux canaux pour la transporter jusqu'aux portes des maisons et des cours des siheyuan 四合院 où ils la vendaient aux habitants à un prix souvent élevé. Profession ancienne, le métier de porteur était très structuré et organisé en clans ou guildes (bang 幫) ${ }^{23}$. À la fin du XIX ${ }^{\mathrm{e}}$ siècle, on en connaît deux : celle du Zhili et celle du Shandong ${ }^{24}$, qui font à la fois office de société mutualiste - en indemnisant les vendeurs en cas d'accident - et de syndicat - en assurant le respect des règles par tous. La profession, très lucrative bien qu'ingrate et épuisante, attirait les vocations, ce qui explique le besoin de réglementer l'activité et de limiter le nombre de porteurs. La concurrence était rude et les disputes fréquentes. Comme les autres métiers du transport, nombreux à Tianjin du fait de la présence d'entrepôts liés au transport du Tribut en grain et des différentes activités commerciales connexes, la profession était dominée par la pègre locale, les fameux Hunhun 混混 ${ }^{25}$ de Tianjin. La plupart d'entre eux faisaient aussi partie des shuihui 水會 (associations de lutte contre le feu).

Ces Hunhun détenaient le pouvoir sur des quartiers entiers de la ville, qui constituaient leur territoire, leur turf; ils y contrôlaient toutes les ressources, y compris l'eau. Les clans de porteurs d'eau s’étaient répartis les rives du Grand Canal, principale source d'approvisionnement, ainsi que les quartiers à desservir sur lesquels ils revendiquaient leurs droits. Leur mainmise sur le commerce de l'eau n'était qu'une des facettes d'un pouvoir à la fois économique et politique ; ils faisaient régner leur loi sans que

${ }^{23}$ D. Spicq, op. cit., p. 65.

${ }^{24}$ Ruth Rogaski, From protecting life to defending the nation, p. 37.

${ }^{25}$ Le terme hun 混 signifie trouble, confus mais aussi mélanger ou gagner sa vie tant bien que mal. Il caractérise assez bien la population flottante qui constituait les hunhun. 
les autorités intervinssent. Les Hunhun ont été la " plaie » de Tianjin pendant tout le $\mathrm{XIX}^{\mathrm{e}}$ siècle, notamment par le racket qu'ils exerçaient à l'occasion mais surtout du fait des incessants combats de rue dont ils étaient les instigateurs.

Après le massacre de Tianjin en $1870^{26}$, le général Zeng Guofan 曾 國藩 (1811-1872), envoyé à Tianjin pour négocier avec les représentants français, rétablit l'ordre et en profite pour faire exécuter de nombreux voyous qui se trouvaient alors emprisonnés sans avoir nécessairement de lien avec les troubles. Par la suite, les gouverneurs généraux du Zhili, Li Hongzhang 李鴻章 (1823-1901) et surtout Yuan Shikai, vont poursuivre l'élimination des Hunhun. Ce sera chose faite à la fin de la décennie 1900, mais la pègre ne disparaît pas pour autant : la « Bande verte » (Qing bang 青幫), société secrète de Shanghai, s’implante à Tianjin à mesure que les Hunhun disparaissent. Cette simultanéité ne semble pas anodine, plusieurs auteurs confirmant d'ailleurs les similitudes entre les deux organisations ${ }^{27}$, notamment au niveau du recrutement de leurs membres.

Les porteurs d'eau disparus, la création des sociétés d'adduction et l'extension du réseau de canalisations donnent naissance à une nouvelle profession : les tenanciers des débits d'eau. De quoi s'agit-il ? Dans la ville chinoise, la majorité de la population n'a pas accès à l'eau à domicile mais va se la procurer dans la rue, à des bornes-fontaine créées à cet effet. Seul

${ }^{26}$ Le massacre de Tianjin désigne les évènements qui ont eu lieu le 21 juin 1870. À la suite d'une tension grandissante entre, d'une part, la mission des prêtres lazaristes et l'orphelinat des sœurs de la Charité, tous ressortissants français protégés par les autorités françaises et, d'autre part, la population de la ville, une foule en colère assassine les prêtres de la mission, les sœurs, une trentaine de convertis chinois, le Consul de France, plusieurs ressortissants français et un couple russe. La mission, l'orphelinat, la cathédrale ainsi que le consulat de France sont également détruits. Ces émeutes antichrétiennes et anti-françaises épargnent le reste de la communauté étrangère de Tianjin. Cf. O. D. Rasmussen, op. cit., p. 45-54.

${ }^{27}$ Gail Hershatter, The Workers of Tianjin, 1900-1949, Stanford : Stanford University Press, 1986, p. 127-129. 
un petit nombre de bâtiments, immeubles et villas, sont directement raccordés au réseau des sociétés d'adduction via des installations sanitaires (robinets, douches et baignoires). Ce type d'installations, coûteux ${ }^{28}$, n'est utilisé que par les plus riches : marchands, compradores, hauts fonctionnaires et seigneurs de la guerre. Dans les concessions, la plupart des étrangers sont directement raccordés au réseau.

En revanche, la grande majorité de la population chinoise utilise les bornes-fontaines, robinets installés sur des bornes directement raccordées aux gros collecteurs qui courent sous les rues. L'entretien et l'utilisation de chaque robinet sont confiés à une personne, le tenancier, qui a passé contrat avec la société d'adduction. Sur chaque robinet est placé un compteur qui enregistre les quantités consommées, facturées au tenancier.

Ceux-ci sont, dans une proportion importante, d'anciens porteurs que les sociétés ont intégrés au nouveau système de distribution. Ils ne semblent pas avoir perdu leurs mauvaises habitudes : tricherie sur les quantités et les prix, ententes entre clans, etc. De 1900 à 1950, la population se plaint régulièrement de leurs agissements. La situation se dégrade particulièrement pendant les années 1930, lorsque la principale société d'adduction, la TNCWC, de concert avec le syndicat des débitants, édicte un règlement en défaveur des clients. Ces derniers se voient alors assigner un vendeur sans pouvoir en changer. Ce règlement limite également la création de nouvelles installations dans certains quartiers. Les raisons d'un tel accord, sans avoir été clairement énoncées, paraissent claires : la TNCWC préfère composer avec les débitants en leur laissant gérer seuls les débits plutôt que d'avoir à les contrôler. Un tel choix a certainement permis à la société de faire des économies aux frais de la population.

La situation s'est encore aggravée sous l’occupation japonaise, notamment à la faveur de l'extension vers des quartiers urbains encore mal

\footnotetext{
${ }^{28}$ La pose et le raccordement des canalisations reliant les installations sanitaires des habitations particulières aux canalisations du réseau situées dans la rue sont aux frais du futur consommateur.
} 
desservis par le réseau d'adduction. Dans les années 1940, de véritables « seigneurs de l'eau » shuiba 水閥 gèrent les débits de quartiers entiers et y font la loi avec la complaisance tacite de la TNCWC.

\section{La fin d'une époque}

La fin de la seconde guerre mondiale et la guerre civile qui suit aggravent les difficultés. La situation à Tianjin est alors assez confuse : la 8 armée de route, communiste, encercle la ville et occupe une partie de la Plaine du Nord au moment de la reddition du Japon le 15 août 1945. Tianjin et sa région semblent prêts à passer sous le contrôle des communistes, mais l'aide américaine permet au Guomindang de renverser la situation à son avantage : sur les 18000 marines américains envoyés fin septembre 1945 à Tanggu 塘沽, autre port de Tianjin sur le Bohai, 4000 d'entre eux parviennent à Tianjin avant les communistes. Les nationalistes peuvent alors réinvestir la ville sans avoir à se battre.

La configuration générale de la ville est alors assez semblable à celle qui a prévalu à la suite de l'insurrection des Boxeurs : une grande partie des bâtiments et de la voirie est détruite, les dégâts et les morts sont nombreux. L'économie est ruinée et la situation sanitaire préoccupante : la disponibilité en eau journalière se situe entre 9 et 19 litres par personne ${ }^{29}$. S’ajoute au problème de la quantité celui de la qualité : l'eau non traitée

\footnotetext{
${ }^{29}$ Le premier chiffre correspond à l'estimation la plus basse donnée par la municipalité qui représente probablement la quantité d'eau effectivement disponible par personne dans les quartiers les plus excentrés de la ville; le second chiffre correspond à l'estimation la plus haute calculée en divisant le nombre d'habitants par le volume de la production d'eau journalière. Ce dernier chiffre est certainement trop élevé si l'on tient compte des pertes dues à la vétusté du réseau de distribution. On considère qu'une personne doit disposer de 15 à 20 litres d'eau potable par jour pour boire, cuisiner et faire sa toilette.
} 
utilisée par les personnes les plus démunies ainsi que celle des puits sont mauvaises. Celle distribuée par la compagnie est de meilleure qualité ; cependant, l'usure et le manque d'entretien des canalisations laissent à penser que l'eau distribuée en fin de réseau, dans les quartiers les plus éloignés du lieu de production, est de qualité médiocre.

En fait, le système d'adduction n'est plus capable d'assurer la production d'une eau potable en quantité et en qualité suffisantes pour la population de la ville. Plusieurs raisons l'expliquent : les équipements sont vieux et abîmés. Les sociétés des eaux, criblées de dettes du fait de l'inflation galopante, ne peuvent investir dans de nouveaux équipements. De plus, face à l'augmentation incessante de la population, la production ne peut suivre la demande, particulièrement dans les nouveaux quartiers situés en périphérie où le nombre de canalisations et de bornes-fontaines est notoirement insuffisant. Enfin le manque de points de distribution a entraîné le développement de monopoles de fait de certains tenanciers qui rackettent la population.

Face à cette situation, le nouveau gouvernement nationaliste place le problème sanitaire au centre de sa politique de reconstruction de la ville. Les abus des vendeurs sont réprimés et ces derniers ont obligation de se faire enregistrer pour exercer leur activité. Peu à peu, le pouvoir de la pègre locale sur les débits est annihilé. On étend le réseau de canalisation aux quartiers périphériques, on augmente le nombre de points de distribution, on réorganise et on fusionne les différentes sociétés. Cette politique volontariste ne sera cependant mise en œuvre que partiellement car, dès le printemps 1946, les combats entre communistes et nationalistes reprennent jusqu'à l'abandon de la ville par l'administration nationaliste en août $1948^{30}$.

Globalement, et malgré sa dépendance à l'égard du pouvoir politique, le système d'adduction de Tianjin, est parvenu pendant la première moitié

${ }^{30}$ Tianjin jianzhi, p. 1403. 
du $\mathrm{XX}^{\mathrm{e}}$ siècle à un niveau technologique satisfaisant, parfois même très comparable à celui des États occidentaux, et a pu fournir aux habitants une eau de qualité en suffisance. Malheureusement, la dégradation de la situation politique, les différents conflits qui ravagent la région de Tianjin de 1937 à 1947 pèsent lourdement sur le système d'approvisionnement. Celuici sortira de cet épisode gravement endommagé.

À partir de 1950, la ressource va être contrôlée par les communistes qui la mettent au service de la population. Dans un pays à nouveau unifié politiquement, ce contrôle redevient avant tout un enjeu économique. Il s’agit pour les autorités de réparer puis d'étendre le système et palier ainsi aux manques les plus criants. Ce n'est en fait qu'après 1949, souvent même à la fin des années 1950, que la plupart des habitants de Tianjin auront enfin l'eau courante à domicile ou à défaut, dans la cour commune située à côté. Force est de constater que les aléas politiques de la période maoïste n’ont pas eu les conséquences négatives sur l'approvisionnement de la ville de ceux de la première moitié du $\mathrm{XX}^{\mathrm{e}}$ siècle.

Aujourd'hui, si l'eau reste à Tianjin une denrée précieuse facilement sujette à la pollution, la régularité de son approvisionnement est devenue un enjeu de stabilité pour le gouvernement. À preuve, les nouveaux projets pharaoniques qui prévoient de dériver une partie de l'eau du Yangzi pour alimenter Pékin et Tianjin. 


\title{
Delphine Spicq
}

\section{Résumé}

Delphine SPICQ : L'eau comme enjeu de pouvoir : l'exemple de Tianjin dans la première moitié du $\mathrm{XX}^{\mathrm{e}}$ siècle

Cet article traite de l'adduction d'eau comme enjeu de pouvoir dans la ville de Tianjin de la fin du $\mathrm{XIX}^{\mathrm{e}}$ siècle au milieu du $\mathrm{XX}^{\mathrm{e}}$ siècle. L'approvisionnement traditionnel était en partie aux mains de la pègre locale qui en contrôlait la distribution. À partir de l'avènement d'un système d'adduction moderne, qui a été mis en place par les Occidentaux dans les concessions étrangères dans un premier temps puis élargi à l'ensemble de la ville après l'épisode des Boxeurs, on a vu plusieurs groupes (les municipalités des concessions étrangères de Tianjin, l'administration de la ville chinoise, les compagnies des eaux, les marchands et industriels, les tenanciers de débits d'eau) se livrer une lutte pour le contrôle de ce nouveau service public et de sa ressource. Les enjeux étaient multiples, essentiellement politiques et économiques, et variaient selon les circonstances. Ce n'est que lorsque la situation politique se stabilise enfin dans la région, après la victoire des communistes sur les nationalistes en 1949, dans un pays à nouveau unifié politiquement, que l'adduction d'eau cesse d'être un enjeu de pouvoir.

\begin{abstract}
Delphine SPICQ : Water as an issue of power: the case of Tianjin in the first half of the twentieth century

This paper deals with the control of the water supply being at stake in Tianjin city from the end of the 19th century to the middle of the 20th century. The traditional way of providing water to the inhabitants of the city was partly in the hands of the local ruffians who controlled the water carriers. The creation of a modern water supply system, first installed by the Westerners only in the city foreign settlements and later widened to the entire city after the Boxers rebellion, resulted in a struggle among different city groups (the settlements municipal councils, the Chinese municipality, the Waterworks companies, the merchants and manufacturers, the water shops keepers) for the control of this public utility and the water resource. Political and economical control was at stake and depended on the circumstances. It is only after the communists victory over the nationalists, when the country being once again politically reunified and the situation in the Tianjin region being stabilized that the water supply ceased to be at stake in the struggle for power of many city groups.
\end{abstract}

